\title{
A new Internal Multi-Model controller for a linear process with an uncertain time delay
}

\author{
TOUZRI MUSTAPHA, NACEUR MONGI, SOUDANI DHAOU \\ Laboratory of Research on Automatic (LARA) \\ National Engineers School of Tunis \\ Tunis, Tunisia \\ touzri.mustapha@gmail.com,m.naceur@ttnet.tn,dhaou.soudani@enit.rnu.tn
}

\begin{abstract}
This document presents a new Internal Multi-Model Command controller design method for a linear system with a limited uncertain time delay. This design method is based on the use of a models collection to approximate the system functioning using Padé approximations; these models are inversed and multiplied by low pass filters in order to obtain a set of controllers to calculate the command value through a fusion procedure. These controllers are obtained by the multiplication of a low pass filters and Models inverses, in order to impose poles and zeros for the considered system and to control the robustness of the command through the filters parameters, which must confirm a compromise between stability and rapidity. In this paper the Multi-Model Command controller design method will be presented through five sections; the first one describes Internal Model Control concepts, the second one describes effects of presence of a time delay on systems dynamics, the third section describes briefly Multi-Model concepts, the fourth section presents the new Internal Multi-Model controller design Method and finally the fifth section presents the obtained results of the new controller design method application for a system with a limited uncertain time delay and the filters parameters variations effects.
\end{abstract}

Keywords- Internal Model Control, Multi-Model approach, Internal Mutli-Model Control, System with a limited uncertain time delay, Padé approximation, Control of systems with time delay.

\section{INTRODUCTION}

Systems including time delay are widely present in industry, due to their dynamics a lot of constraints make the control of such systems difficult [5].

For this reason control of time delay became one of the most attractive research domain [10]; that's why many command structure are used to surpass these constraints in order to obtain an acceptable process behavior such as Internal Model Control which will be combined with a Multi-Model approach to control a SISO process with a limited variable time delay.

The aim of this research is to introduce a new controller design method based on Internal Model control adopted with Multi-Model concepts in order to surpass constraints imposed by the variable time delay and to obtain a robust process behavior.

This document describe a new Internal Model Controller design method combined with a Multi-Model approach in five sections; the first one describe Internal Model Control
Concepts, the second one describe constraints due to time delay and utility of using Padé approximations, the third section present Multi-Model concepts, the fourth section present the new Internal Model Controller design method and the last section present the obtained results of the new controller design method applied for a linear system with an uncertain time delay.

\section{IMC DESCRIPTION}

Many command structures were developed using the feedback concept; which uses mathematical approaches to solve problems related to processes command, these approach was implemented by the apparition of the first calculator [4]. Internal Model Control, noted as IMC, uses feedback concept and uses the robust command characteristics, which can ensure an acceptable degree of performance even on the presence of parameters uncertainties and/or modelisations errors [1]. The basic structure of an IMC command is composed by the process compared to its model, and a controller as it shown on figure 1 [7].

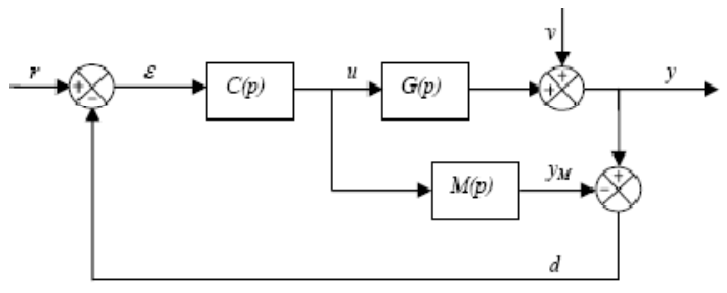

Figure 1. Basic IMC structure

Where $\mathrm{C}(\mathrm{p})$ represents the IMC controller, $\mathrm{G}(\mathrm{p})$ the process and $M$ the model of the process which is an approximation of the plant $G(p)$. This command structure applies the command signal $u$ for both of the process $G(p)$ and its model $M(p), d$ is a disturbance signal which attacks the output directly and $r$ is the reference signal, the output signal of the plant is compared to the set point signal in order to minimize the error between the reference and the output.

Internal model Control is one of the most popular command structure used for its simplicity and its robustness; this control method gives the possibility to realize a perfect set point tracking in the case of the use of a controller similar to the inverse of the model [7].

Achieving the inverse of the model is the main problem associated to this command structure, because of the 
denominator order generally greater than the numerator on the model expression or the presence of time delay or/and instable zeros [2].

\section{CONSTRAINTS IMPOSED BY TIME DELAY}

Systems with delays are found in many industrial processes. Time delay presence is due to many factors such as transfer of information, energy or chemical reactions $[5,10]$. Then presence of delays makes system analysis and controller design more complex [10], due to the time delay effects on the system behavior which imposes many constraints on system command. Delays constraints may cause instability and deterioration on the system performances especially on closed loop.

Time delay also can cause a lag on the system phase especially for its high values [5] and for elevated frequency, which can be the reason of closed loop system performances deterioration or instability. Time delay presence make also the effect of the disturbances not felt until a considerable time has elapsed, the effect of the control action takes some time to be felt in the controlled variable and the control action that is applied based on the actual error tries to correct a situation that originated some time before [5].

Using the IMC structure, the associated controller can be used as the inverse of the process model; however in the case of presence of a time delay gives a prediction system, when the inverse is calculated, making the realization of this type of systems difficult. For this purpose a Padé approximation is used to surpass these constraints, of inversion and realization, and giving a rational representation for the process making possible the inversion of the process model. But this approximation gives an alternative to modelisations errors which can destroy the system performances and drive its behavior to the instability, to face this constraint we use on our command structure two Padé order approximation; a first order, and a second order approximation to decrease the effects of modelisations errors by elevating the approximation order to have models that can behave as the original process for high frequency, then higher order approximations gives same behavior of the plant for more higher frequency. The obtained models using Padé approximation will be used to calculate the Internal Multi-Model Command controller, and section IV objective is to give a command approach that can solve the realization problem of the IMC controller.

\section{MUlTI-MODEL COMMAND CONCEPTS}

This section describes briefly concepts of a Multi-Model controller. Multi-Model concepts are introduced on modelisation for the non linear systems and the not well known dynamics systems; the main purpose of using Multi-Model approach is to obtain the best representation for the system dynamics by calculating validity coefficients and then realizing commutation or fusion between these models.

These concepts were generalized for the design of controller that uses different commands at the same time and selects the best one for the process using many parameters and procedure or algorithms allowing an optimal behavior for the considered process [3]. Multi-Model approach is based on a collection of models that represents system dynamics, several operating point for non linear system, then calculates models validities and the fusion of these collected data using specified methods [3].

Then a Multi-Model controller uses these concepts in order to obtain a robust command that ensures optimal performances for the considered process.

In fact Multi-Model controller uses a collection of models and their validities to calculate the best command for the process by using an algorithm for the fusion between these data.

For this purpose many fusion method were developed to satisfy the Multi-Model controller requirements [3] were the designer uses different algorithms to obtain the best command that allow a robust behavior for the process.

In this document we will use a Multi-Model controller combined with Internal Model Control concepts which will be generalized as Internal Multi-Model Controller that uses commutation between controllers as a fusion method for the command of a linear system with an uncertain limited time delay which will be described on the next section.

\section{InTERnAl Multi-MODEL CONTROLLER DESIGN PROCEDURE}

This section describes the design method of the new Internal Multi-Model Controller which is based on the use of different low pass filters associated to each model of the considered process.

In fact the new Internal Multi-Model controller design method is a combination between, the Internal Model Controller design method described on [8,9] and Multi-Model approach; this new design method can be described by this figure:

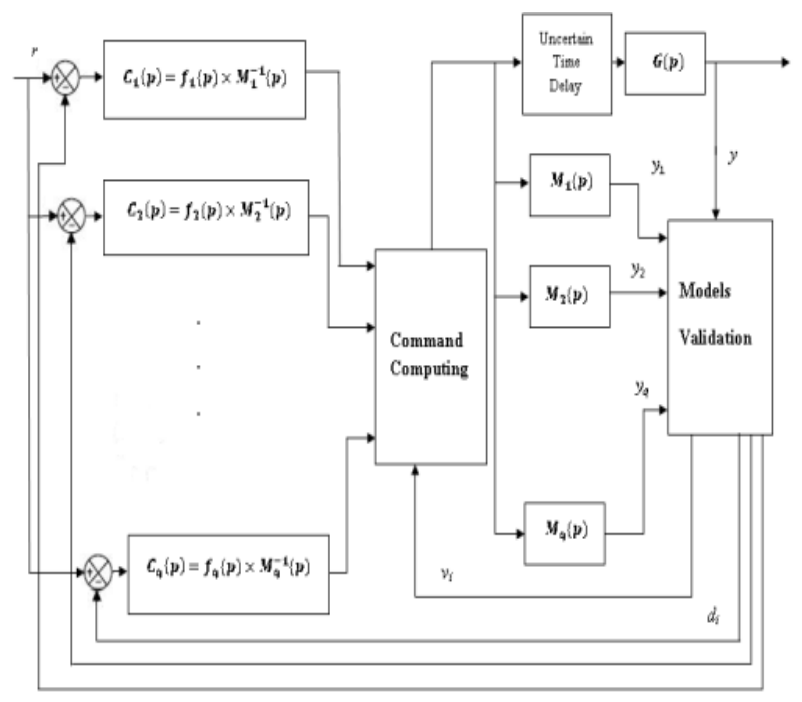

Figure 2. Internal Multi-Model Control structure

This command structure applies the same command for the process and its models $M_{1}(p) \ldots M_{q}(p)$, then calculates validity coefficients $v_{i}$ which will be used to compute the command value that will be selected from one of the associated controllers $\mathrm{C}_{1}(\mathrm{p}) \ldots \mathrm{C}_{\mathrm{q}}(\mathrm{p})$ that receives the difference between 
the reference and the outputs of the used models in order to minimize the errors. Then a fusion strategy is used to calculate the command value, its main idea is to quantify every partial command contribution $\mathrm{u}_{\mathrm{i}}(\mathrm{t})$ to the global command $\mathrm{u}(\mathrm{t})$; for this purpose, we use validity coefficients that approve the closest model to the process [7].

These validity coefficients $v_{i}(t)$ are inversely proportional to the distance $d_{i}(t)=y(t)-y_{i}(t)$ where $y$ is the process output and $y_{i}(t)$ is the model $M_{i}(t)$ output.

Then it will be compulsory to satisfy this condition :" The more the model $M_{i}(t)$ output is closest to the process output the more the contribution of the partial command $u_{i}(t)$ in the global command $\mathrm{u}(\mathrm{t})$ " [3]; then validation coefficient will be calculated using this expression [7]:

$$
\mathrm{v}_{\mathrm{i}}(\mathrm{t})=\frac{\left\|\frac{1}{\mathrm{~d}_{\mathrm{i}}}\right\|}{\sum_{\mathrm{j}=1}^{\mathrm{q}}\left\|\frac{1}{\mathrm{~d}_{\mathrm{j}}}\right\|}
$$

and the global command expression will use these validity coefficients and the partial command $\mathrm{u}_{\mathrm{i}}(\mathrm{t})$, then the global command will be expressed by [7]:

$$
u(t)=\sum_{i=1}^{q} v_{i}(t) u_{i}(t)
$$

Then every partial command $u_{i}(t)$ is the output of the controllers $C_{i}(p)$ realized by multiplication of a low pass filter $f_{i}(p)$ and the inverse of the model $M_{i}(p)$ and can be written:

$$
\mathrm{C}_{\mathrm{i}}(\mathrm{p})=\mathrm{f}_{\mathrm{i}}(\mathrm{p}) \times \mathrm{M}_{\mathrm{i}}^{-1}(\mathrm{p})
$$

$(i=1 \ldots q)$, where $f_{i}(p)$ is associated to the model inverse $M_{i}(p)$ and each filter $f_{i}(p)$ contains instable zeros that can appear on the process model expression $\mathrm{M}_{\mathrm{i}}(\mathrm{p})$.

Then for each Controller $C_{i}(p)$ the used filter $f_{i}(p)$ can be written on this form [6]:

$$
f_{i}(p)=\frac{\sum_{j=0}^{m} \beta_{j} p^{j}}{(1+\alpha p)^{n}}
$$

Where:

$\sum_{\mathrm{j}=0}^{\mathrm{m}} \beta_{\mathrm{j}} \mathrm{p}^{\mathrm{j}}$ is the instable zeros that can appear on the process model

$\mathrm{n}$ : is a natural integer chosen to make the controller $\mathrm{C}_{\mathrm{i}}(\mathrm{p})$ proper.

:is a float used to adjust the performance of the controlled process.

And each controller $\mathrm{C}_{\mathrm{i}}(\mathrm{p})$ can be computed using this method:

$$
\mathrm{M}_{\mathrm{i}}(\mathrm{p})=\frac{\mathrm{N}_{\mathrm{ZS}}(\mathrm{p}) \times \mathrm{N}_{\mathrm{ZI}}(\mathrm{p})}{\mathrm{D}(\mathrm{p})}
$$

$$
\begin{array}{r}
M_{i}^{-1}(p)=\frac{D(p)}{N_{Z S}(p) \times N_{Z I}(p)} \\
f_{i}(p)=\frac{N_{Z I}(p)}{(1+\alpha p)^{n}} \\
C_{i}(p)=f_{i}(p) \times M_{i}^{-1}(p) \\
=\frac{D(p)}{N_{Z S}(p) \times(1+\alpha p)^{n}}
\end{array}
$$

Where:

$\mathrm{N}_{\mathrm{ZS}}(\mathrm{p})$ : is the stable zeros on the numerator of the process model $M_{i}(p)$.

$\mathrm{N}_{\mathrm{ZI}}(\mathrm{p})$ : is the instable zeros that can be present on the process model $M_{i}(p)$.

$D(p)$ : represent the denominator of the process model $M_{i}(p)$.

$\mathrm{n}$ : is a natural integer chosen to make the controller proper $\mathrm{C}_{\mathrm{i}}(\mathrm{p})$.

$\alpha$ : is a float used to adjust the system performances.

In this command structure, the aim of using a Multi-Model approach is to surpass constraints due to the uncertain time delay, besides the combination between Multi-Model concepts and Internal Model Control is to ensure the robustness of the command in spite of disturbance presence and modelisation errors. In fact this command structure allow us to impose the poles and zeros of the process, such as the filter order or the filter pole or the filter zeros and to use the closest model to the process when computing the command, these characteristics allow the designer to control the robustness level of the command by choosing parameters that ensures the best compromise between robustness and rapidity.

Then for each filter the choice of its pole must confirm an acceptable compromise between stability and rapidity, the filter pole can be chosen using these recommendations:

if $\alpha=0$ the system response will be $\mathrm{H}_{2}$ - optimal [6]

if $\alpha$ is chosen greater than the poles of the controlled system the filter dynamics will dominates the closed loop response of the system [6]

and if $\alpha$ is chosen inferior to the poles of the system the filter effect will not dominates the closed loop response of the system [6].

Then the parameter filter $\alpha$ allow us to control the speed of the closed loop response and that the adjusting of $\alpha$ is the same as adjusting the speed of the closed loop response [6].

\section{OBTAINED RESULTS FOR A VARIABLE TIME DELAY FIRST ORDER SYSTEM USING DIFFERENT PADÉ APPROXIMATION}

This section shows the obtained results of the simulations of a linear process with variable time delay functioning; using the new command structure, the considered process is characterized by this expression: $G(p)=\frac{e^{-(\tau+\delta \tau)}}{1+5 p}$ where $\tau+\delta \tau$ is a limited uncertain time delay and $(\tau+\delta \tau \leq 4)$ were $\tau=2$ 
and $\delta \tau$ is not known, then the variation of the uncertain time delay $\tau+\delta \tau$ is described by figure 3 .

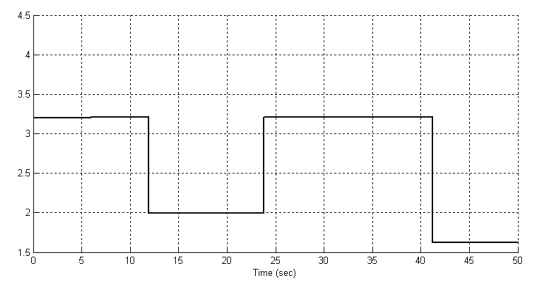

Figure .3 Time delay variation

This time delay will be estimated using four models each one of them will be calculated using a fixed value $\tau_{\mathrm{i}}=\tau+\delta \tau_{\mathrm{i}}$ for $\mathrm{i}=1 \ldots 4$ then $\tau_{1}=1 \mathrm{~s}, \tau_{2}=2 \mathrm{~s}, \tau_{3}=3 \mathrm{~s}$ and $\tau_{4}=4 \mathrm{~s}$, then the considered process is estimated to four systems with a fixed time delay expressed by:

$$
\begin{aligned}
& \mathrm{G}_{1}(\mathrm{p})=\frac{\mathrm{e}^{-\mathrm{p}}}{1+5 \mathrm{p}} \quad, \quad \mathrm{G}_{2}(\mathrm{p})=\frac{\mathrm{e}^{-2 \mathrm{p}}}{1+5 \mathrm{p}} \quad, \quad \mathrm{G}_{3}(\mathrm{p})=\frac{\mathrm{e}^{-3 \mathrm{p}}}{1+5 \mathrm{p}} \quad \text { and } \\
& \mathrm{G}_{4}(\mathrm{p})=\frac{\mathrm{e}^{-4 \mathrm{p}}}{1+5 \mathrm{p}} .
\end{aligned}
$$

Then for each one of these transfer functions a first order and a second order Padé approximations will be used to obtain models expression. Then this section contains two subsections, the first one shows the obtained results of using a first order Padé approximation to calculate system models and the second one shows the obtained results of using a second order Padé approximation to calculate system models.

\section{A. Obtained results using a first order Padé approximation}

The considered process is estimated to four systems with a fixed time delay, then a first order Padé approximation will be used to calculate system models described by:

$$
\begin{aligned}
& \mathrm{M}_{1}(\mathrm{p})=\frac{1-\frac{\mathrm{p}}{2}}{1+5.5 \mathrm{p}+2.5 \mathrm{p}^{2}} \text { for } \tau=1 \mathrm{~s}, \\
& \mathrm{M}_{2}(\mathrm{p})=\frac{1-\mathrm{p}}{1+6 \mathrm{p}+5 \mathrm{p}^{2}} \text { for } \tau=2 \mathrm{~s}, \\
& \mathrm{M}_{3}(\mathrm{p})=\frac{1-\frac{3}{2} \mathrm{p}}{1+6.5 \mathrm{p}+7.5 \mathrm{p}^{2}} \text { for } \tau=3 \mathrm{~s}, \\
& \text { and } \mathrm{M}_{4}(\mathrm{p})=\frac{1-2 \mathrm{p}}{1+7 \mathrm{p}+10 \mathrm{p}^{2}} \text { for } \tau=4 \mathrm{~s}
\end{aligned}
$$

Then a filter $f_{i}(p)$ will be associated to each model $M_{i}(p)$; which contains instable zeros of these models that will be eliminated from the controller $\mathrm{C}_{\mathrm{i}}(\mathrm{p})$ expression which will be calculated as it was described on the previous section by multiplying the models inverse and low pass filters.

Then the used filters and the calculated controllers are expressed on the table below:
TABLE I. LIST OF FILTERS AND CONTROLLERS FOR EACH PROCESS MODEL

\begin{tabular}{|l|c|c|}
\hline$M_{i}(p)$ & $f_{i}(p)$ & $C_{i}(p)=f_{i}(p) \times M_{i}^{-1}(p)$ \\
\hline$M_{1}(p)$ & $f_{1}(p)=\frac{1-\frac{p}{2}}{(1+\alpha p)^{2}}$ & $C_{1}(p)=\frac{1+5.5 p+2.5 p^{2}}{(1+\alpha p)^{2}}$ \\
\hline$M_{2}(p)$ & $f_{2}(p)=\frac{1-p}{(1+\alpha p)^{2}}$ & $C_{2}(p)=\frac{1+6 p+5 p^{2}}{(1+\alpha p)^{2}}$ \\
\hline$M_{3}(p)$ & $f_{3}(p)=\frac{1-\frac{3}{2} p}{(1+\alpha p)^{2}}$ & $C_{3}(p)=\frac{1+6.5 p+7.5 p^{2}}{(1+\alpha p)^{2}}$ \\
\hline$M_{4}(p)$ & $f_{4}(p)=\frac{1-2 p}{(1+\alpha p)^{2}}$ & $C_{4}(p)=\frac{1+7 p+10 p^{2}}{(1+\alpha p)^{2}}$ \\
\hline
\end{tabular}

The simulations results will be shown on figure 4 where the controllers are used for different value of the filters parameter $\alpha$ to show the effects on the process behavior.

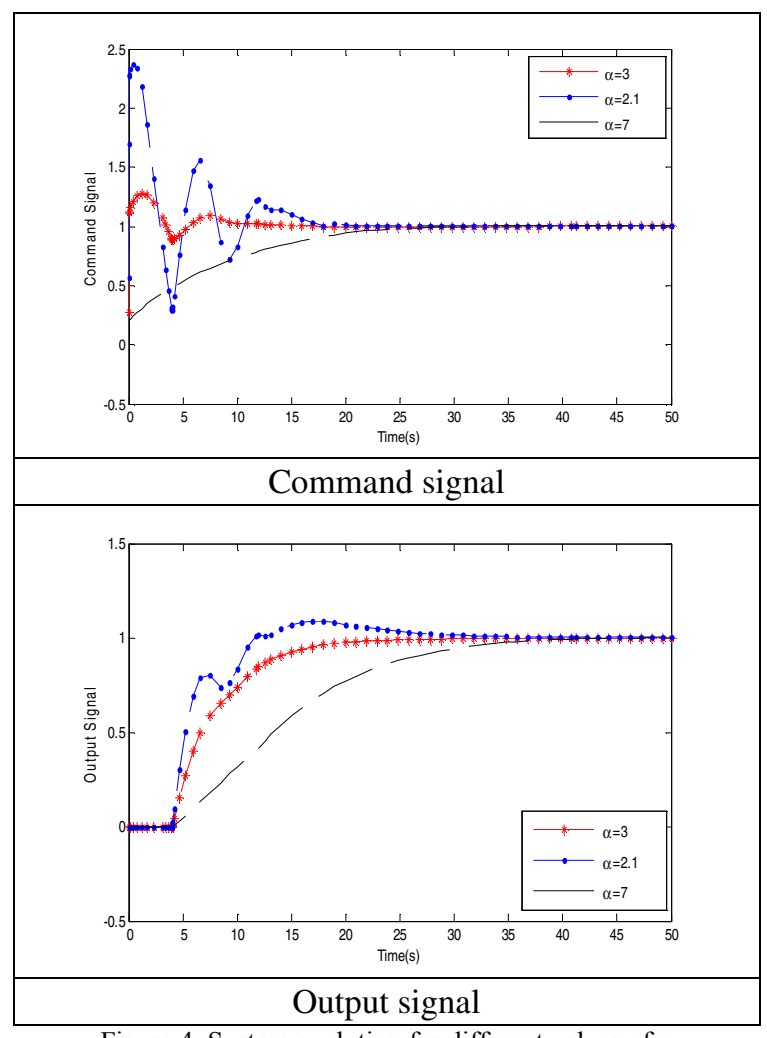

Figure 4. System evolution for different values of $\alpha$

These simulations show that the new used command gives a robust behavior characterized by the fast set-point tracking even on the presence of the uncertain time delay; this can be seen on the system step response for $\alpha=3$ where the command signal and the output signal are smoothly perusing the setpoint, however the use of much high values of $\alpha$ can make the system response slow and not robust such is the case of the use 
of $\alpha=7$ where the system response is slow and does not confirm conditions of a robust behavior where the system obey for a compromise between stability and rapidity; also the use of smaller values of $\alpha$ that are closer to the system poles can lead the system behavior to instability such is the case of $\alpha=2.1$, where the filter pole is chosen smaller then a non dominant model pole $\mathrm{p}=-2$ (for the value of $\tau=1 \mathrm{~s}$ ) where its contribution on the command could not impose its dynamics which is due to the used fusion procedure that improve the closest model to the process on the command computing, in this case the system step response presents a peak and dumped oscillations on the command signal and oscillations on the output signal.

\section{B. Obtained results using a second order Padé approximation}

As it was done on the previous section. The process is estimated to four systems with a fixed time delay then a second order Padé approximation will be used to compute system models $\mathrm{M}_{\mathrm{i}}(\mathrm{p})$ described by:

$$
\begin{aligned}
& M_{1}(p)=\frac{1-\frac{p}{2}+\frac{p^{2}}{12}}{1+5.5 p+\frac{31}{12} p^{2}+\frac{5}{12} p^{3}} \text { for } \tau=1 s, \\
& M_{2}(p)=\frac{1-p+\frac{p^{2}}{3}}{1+6 p+5.33 p^{2}+\frac{5}{3} p^{3}} \text { for } \tau=2 s, \\
& M_{3}(p)=\frac{1-\frac{3}{2} p+\frac{3}{4} p^{2}}{1+6.5 p+8.25 p^{2}+\frac{15}{4} p^{3}} \text { for } \tau=3 s, \\
& \text { and } M_{4}(p)=\frac{1-2 p+\frac{4}{3} p^{2}}{1+7 p+\frac{44}{3} p^{2}+\frac{20}{3} p^{3}} \text { for } \tau=4 s
\end{aligned}
$$

After this a filter $f_{i}(p)$ will be associated to each model $M_{i}(p)$; which contains instable zeros of these models that will be removed from the controller $\mathrm{C}_{\mathrm{i}}(\mathrm{p})$; expression, the used filters are described by:

$f_{1}(p)=\frac{1-\frac{p}{2}+\frac{p^{2}}{12}}{(1+\alpha p)^{3}}$ for $M_{1}(p), f_{2}(p)=\frac{1-p+\frac{p^{2}}{3}}{(1+\alpha p)^{3}}$ for $M_{2}(p)$,

$f_{3}(p)=\frac{1-\frac{3}{2} p+\frac{3}{4} p^{2}}{(1+\alpha p)^{3}}$ for $M_{3}(p)$, and $f_{4}(p)=\frac{1-2 p+\frac{4}{3} p^{2}}{(1+\alpha p)^{3}}$ for

$\mathrm{M}_{4}(\mathrm{p})$.

Then the controllers can be computed as it is described on section IV, and are described by: $C_{i}(p)=f_{i}(p) \times M_{i}^{-1}(p)$ for $\mathrm{i}=1,2,3$ and 4 ; where

$$
\mathrm{C}_{1}(\mathrm{p})=\frac{1+5.5 \mathrm{p}+\frac{31}{12} \mathrm{p}^{2}+\frac{5}{12} \mathrm{p}^{3}}{(1+\alpha p)^{3}}
$$

$\mathrm{C}_{2}(\mathrm{p})=\frac{1+6 \mathrm{p}+5.33 \mathrm{p}^{2}+\frac{5}{3} \mathrm{p}^{3}}{(1+\alpha \mathrm{p})^{3}}$

$\mathrm{C}_{3}(\mathrm{p})=\frac{1+6.5 \mathrm{p}+8.25 \mathrm{p}^{2}+\frac{15}{4} \mathrm{p}^{3}}{(1+\alpha \mathrm{p})^{3}}$,

and $\mathrm{C}_{4}(\mathrm{p})=\frac{1+7 \mathrm{p}+\frac{44}{3} \mathrm{p}^{2}+\frac{20}{3} \mathrm{p}^{3}}{(1+\alpha \mathrm{p})^{3}}$.

The simulations results will be shown on figure 5 where the controllers are used for different value of the filters parameter $\alpha$ to show the effects of its variation on the process behavior.

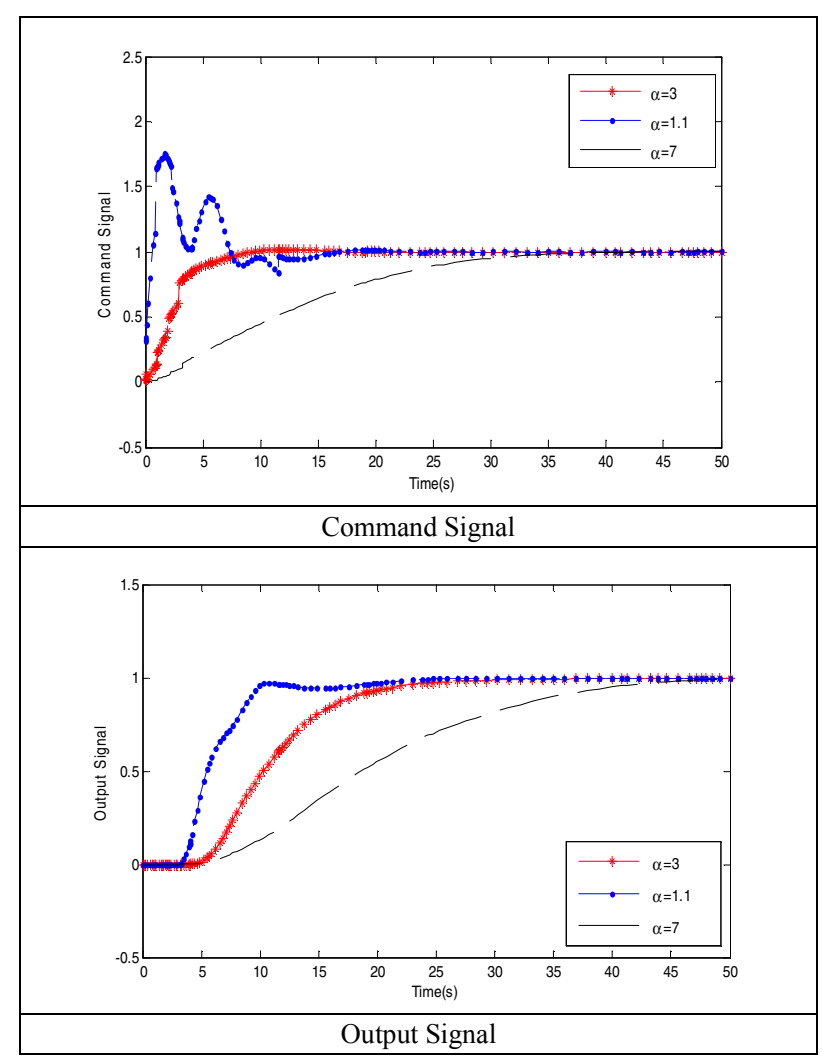

Figure 5. System evolution for different values of $\alpha$

It can be seen that the new command structure that uses validation coefficient on the command computing; gives a robust behavior characterized by the fast set-point tracking even on the presence of the uncertain time delay; it is remarkable also that the use of high order Padé approximation gives the opportunity to use a filter pole closer to the models poles such is the case of using $\alpha=1.1$ where the system presents an acceptable behavior for output signal and presents a peak and dumped oscillations on the command signal that damage the system component, for this purpose we use a higher values for the filter pole $\alpha$ to make the system behavior more stable such is the case of $\alpha=3$ where the process presents a fast and smooth set-point tracking which is remarkable on the command signal and the output signal that tracks the reference smoothly. However the use of much higher values for the filter 
pole can make the system response slow and not robust such is the case of using $\alpha=7$ where the process output is slow and not robust which is not confirm for a robust command characteristics.

\section{CONCLUSION}

In this work a new command structure were developed for the control of linear system with an uncertain time delay. This command structure is based on the combination of MultiModel concepts and Internal Model Control in order to obtain a robust system behavior. Unfortunately a collection of models, are calculated using Padé approximations, which is used to approximate the process expression and their inverse will be associated to a collection of low pass filters; in order to obtain a set of controllers, that gives a collection of partial commands and a collection of validity coefficients which are used to calculate the global command value in order to obtain a command that ensures a robust behavior.

This new Internal Multi-Model Command design method gives interesting results for a linear process with a limited uncertain time delay and shows a robust behavior; and allow the designer to control the robustness level of the command through the variation of the filters parameters in order to obtain an acceptable compromise between stability and rapidity which is the main advantage of this command structure.

\section{REFERENCES}

[1] ACKERMANN J., "Robust control: Systems with uncertain physical parameters", Springer-Verlag, New York, 1993.

[2] BROWN M.D., LIGHTBODY G., and IRWIN G.W., "Nonlinear internal model control using local networks", IEE Proceedings-Control Theory and Applications, Vol. 144, pp. 505-514, 1997.

[3] DELMOTE F., " Analyse multimodèle", Thèse de Doctorat, USTL, Lille, 1997.

[4] GARCIA C.E. et MORARI M.," Internal Model Control 1- A unifying review and some results", Ind. Eng. Chem. Process Des. Dev., Vol. 21, pp. 403-411, 1982.

[5] J.E.NORMEY-RICO et E.F CAMACHO, "Control of Dead-time Processes", Springer-Verlag London,2007.

[6] MORARI M. et ZAFIROU E., "Robust process control ", Prentice Hall, USA, 1989.

[7] NACEUR M. , "Sur la commande par modèle interne des systèmes dynamiques continus et échantillonnés", Thèse de Doctorat, ENIT, Tunis, 2008.

[8] TOUZRI M. , NACEUR M. , SOUDANI D. , "A New IMC Controller Design Method Using a Low Pass Filter and Variation Effects of Its order", 2nd International Conference on System and Control, ICSC'2012, Marrakech MAROCCO.

[9] TOUZRI M. , NACEUR M. , SOUDANI D. , "A new design method of an imc controller for a second order system with time delay and an instable zero", Twelfth International conference on Science and Techniques of Automatic Control \& computer engineering, STA'2011, Sousse TUNISIA, 2011, pp 123-133.

[10] ZHONG Qing-Chang "Robust control of time-delay systems", Springer-Verlag London 2006. 